\title{
Joint position statement on the management of mesh-related complications for the FPMRS specialist
}

\author{
Developed by the Joint Writing Group of the American Urogynecologic Society \\ and the International Urogynecological Association
}

Published online: 7 February 2020

(C) American Urogynecologic Society and International Urogynecological Association 2020

\begin{abstract}
The scientific approach to categorizing mesh complications and optimal methods to address them have been complicated by the rapid proliferation and evolution of materials and techniques that have been used over the past 20 years in surgical treatment of pelvic floor disorders. In addition, terminology used to diagnose and categorize mesh complications and the descriptions of surgical procedures to manage them have been adopted inconsistently, further hampering the development of a collective experience with a standardized lexicon. Finally, much of the high-quality data on management of mesh complications is based on materials that are rarely used or not commercially available today.

Women experiencing mesh complications need to be heard and should have access to resources and providers who are most able to help. Many women require multiple procedures to address their mesh complications, and for some of these patients, relief is incomplete. We should strive to optimize the treatment at the initial diagnosis of a mesh-related complication.

This Position Statement has 4 goals:

1. Using the best and most relevant evidence available, provide guidance for the FPMRS subspecialist caring for patients who may be experiencing mesh complications

2: Provide an algorithm outlining treatment choices for patients with mesh-related complications that can be used as a platform for shared decision making in the treatment of these complications

3: Identify and prioritize gaps in evidence concerning specific mesh complications and their treatments

4: Identify provider and health facility characteristics that may optimize the outcomes of treatments for these complications
\end{abstract}

Keywords pelvic floor disorder · mesh complications

The AAGL endorses this document. The Society of Gynecologic Surgeons supports this document. Individual contributors are noted in the Acknowledgments section.

This report is being published concurrently in Female Pelvic Medicine and Reconstructive Surgery and in International Urogynecology Journal. The report is identical except for minor stylistic and spelling differences in keeping with each journal's style. Citations from any of the two journals can be used when citing this article.

Disclaimer: This Position Statement was developed by a joint writing group between the American Urogynecologic Society and the International Urogynecological Association. This document reflects clinical and scientific understanding as of the date issued and is subject to change. The information should not be construed as dictating an exclusive course of treatment or procedure to be followed.

Correspondence: Charles R. Rardin, MD, Division of Urogynecology and Reconstructive Pelvic Surgery, Department of Obstetrics and Gynecology, Alpert Medical School of Brown University, 101 Plain Street, 5th Floor, Providence, RI 02903, USA.

Email: crardin@wihri.org
Providers caring for women with pelvic floor disorders are aware that surgery has attendant risks that counterbalance the benefits. Decisions concerning the balance of risks and benefits should be made in collaboration with each patient. In recent decades, frustration with surgical failures has prompted development of materials and techniques using synthetic mesh. In some circumstances, these techniques seem to confer durability, but the implants themselves introduce unique risks. The 2011 Update to the Food and Drug Administration's Public Health Notification regarding use of transvaginal mesh (TVM) stated that these complications are not rare and can be difficult to treat.

The scientific approach to categorizing mesh complications and optimal methods to address them have been complicated by the rapid proliferation and evolution of materials and techniques that have been used over the past 20 years in surgical treatment of 
pelvic floor disorders. In addition, terminology used to diagnose and categorize mesh complications and the descriptions of surgical procedures to manage them have been adopted inconsistently, further hampering the development of a collective experience with a standardized lexicon. Finally, much of the high-quality data on management of mesh complications is based on materials that are rarely used or not commercially available today.

Women experiencing mesh complications need to be heard and should have access to resources and providers who are most able to help. Many women require multiple procedures to address their mesh complications, and for some of these patients, relief is incomplete. We should strive to optimize the treatment at the initial diagnosis of a mesh-related complication.

This document is a companion to the Committee Opinion released jointly by the American College of Obstetricians and Gynecologists and the American Urogynecologic Society (AUGS), which was a reference for the OB/Gyn specialist [1]. This document has been developed by AUGS and the International Urogynecological Association (IUGA) as a resource for the subspecialist in female pelvic medicine and reconstructive surgery (FPMRS). A call for applications to the writing group was issued to the AUGS and IUGA membership. Applications, including conflicts of interest, were reviewed and the writing group chair was identified. The members of the writing group were chosen by invitation to ensure a wide range of expertise. We emphasize that the level of supporting evidence does not allow us to consider this a true guideline, but is an assessment of the state of the art at the moment and will allow a provider to give simple context to a patient who may be experiencing a complication.

This Position Statement has 4 goals:

1. Using the best and most relevant evidence available, provide guidance for the FPMRS subspecialist caring for patients who may be experiencing mesh complications

2. Provide an algorithm outlining treatment choices for patients with mesh-related complications that can be used as a platform for shared decision making in the treatment of these complications

3. Identify and prioritize gaps in evidence concerning specific mesh complications and their treatments

4. Identify provider and health facility characteristics that may optimize the outcomes of treatments for these complications

\section{Restrictions on the focus of this document}

This document does not explore risk factors for mesh complications; patient selection, instruction, or surgical techniques that may reduce the likelihood of such complications are beyond its scope. It is intended to provide guidance to a subspecialist provider at the point of recognition of a potential complication and to provide a platform for shared decision making with the patient. Similarly, for the purposes of this document, voiding dysfunction after synthetic midurethral slings (MUSs) and recurrence of stress urinary incontinence (SUI) or prolapse after mesh-based repair are not considered true mesh complications; they reflect functional outcomes. These conditions are also relevant to nonmesh procedures, and their management is not addressed here.

Finally, issues related to sexual function after mesh-based surgeries are extremely complex. This document addresses postoperative sexual dysfunction only as it relates to meshrelated pain for the woman or for her partner.

\section{Expectations of FPMRS subspecialists in diagnosis and initial management of mesh complications (Adapted from the UK National Institute for Health and Care Excellence [NICE] Guidance Document, 2019)}

Upon recognition of the possibility of a mesh-related complication, the FPMRS subspecialist should do the following:

- Obtain all necessary historical information, including the specifics of implanted devices and onset of symptoms relative to the index procedure. It is incumbent on the subspecialist who may propose surgical intervention to have an adequate understanding of the index procedure. This understanding is imperative whether or not the index procedure was part of the subspecialist's training, or whether or not it remains commercially available.

- Perform a pelvic examination to assess for mesh exposure, to localize the pain anatomically, and assess its relationship to the location of the implanted mesh [2]. Generally, if the pain is not localized and elicited during the pelvic examination, one should be cautious about attributing the pain to the mesh implant. Similarly, pelvic neurologic examination can help distinguish between local effects and those resulting from nerve entrapment or impingement.

- Office-based pelvic examinations are the first line of evaluation. However, in some cases, including intolerance of an adequate pelvic examination, examination under anesthesia may offer diagnostic benefit.

- Additional diagnostic procedures should be considered as determined by the specifics of the patient, the index procedure/implanted device, and symptoms. Cystoscopy, vaginoscopy, and digital rectal examination should be performed as appropriate. It has been suggested that imaging modalities such as endoluminal or transperineal ultrasound or magnetic resonance imaging are beneficial for diagnosing mesh complications and mapping surgical treatment strategies. 
- It is incumbent on the subspecialist to develop as complete a diagnostic understanding as possible before embarking on surgical treatment. The provider should have an appropriate index of suspicion to engage the appropriate consultative assistance (eg, colorectal or neurosurgery) ahead of time. It is worth considering that, during surgical treatment of vaginal mesh exposures, unexpected mesh exposures in the bladder or bowel are encountered in $3 \%$ of cases [3].

\section{Definitions and classifications}

Every effort has been made to align the nomenclature in this Statement with terms outlined by international FPMRS organizations. We therefore use the terminology laid out by IUGAICS for use in diagnosis, management, and outcomes assessment of mesh-related complications [4].

For the purpose of this statement and its focus on the management of mesh complications after they have been identified, terms such as "extrusion," "contraction," "separation," "dehiscence," and "perforation" imply either a causality or a time course that is beyond the scope of this document. The term "mesh erosion" has long been used to describe a variety of mesh-related complications, but it has been used inconsistently. In some circles, it is meant to refer to mesh encountered in the bladder or bowel, but the term itself lacks specificity. In addition, the term "erosion" is not included in the IUGA-ICS terminology document. Accordingly, this document does not use the term "erosion."

Therefore, "mesh exposure" with a description of where that exposure is located is used (eg, "vaginal mesh exposure" or "rectal mesh exposure"). To decrease confusion, we recommend that the anatomic location of the exposed mesh always be used along with the term "mesh exposure."

We note that there may be relevance for terms such as erosion, contraction, protrusion, perforation, banding, or prominence in documents investigating causes, risk factors, techniques, or natural history of mesh-related complications, but these will not be used in this document, which is focused only on mesh complication management.

Proper classification of mesh complications is imperative for development of treatment algorithms and decision-support tools. The IUGA ICS Classification System for Mesh Complications was developed in an effort to improve this process. An online calculator to assist in the classification of mesh complications is available at https://www.ics.org/complication; FPMRS providers for women with mesh-related complications are encouraged to execute due diligence in the diagnosis and to record the classification of these complications.

The recommendations in this Position Statement are informed by a thorough review of current literature. However, the heterogeneity and/or lack of detail in the nature and extent of the surgical interventions for mesh-related complications in the published literature present considerable challenges for
Table 1 Surgical procedures for the treatment of mesh complications

Mesh revision

Either no mesh is removed (eg, dissecting and primarily closing vaginal epithelium), or a small edge of mesh is removed such that the structural integrity of the implant is left intact.

Partial vaginal mesh excision

A segment/component of the mesh is removed or transected, such that the structural integrity of the implant is altered.

Complete vaginal mesh excision

The entirety of the mesh that is in contact with the vagina is excised

Extravaginal mesh excision

This involves removal of segments or components of mesh beyond, or not in contact with, the vagina. Note the following:

- Because of the wide variation of devices and approaches, this category

should include additional description of which mesh segments were removed.

- This term should be used in addition to any relevant vaginal mesh excision, if performed.

Total mesh excision

The surgical goal is the removal of $100 \%$ of the implant (extirpation).

synthesizing current knowledge. With these challenges in mind, we propose that surgical procedures for treatment of mesh complications be categorized as described in Table 1.

See Figs. 1, 2 and 3 for examples of retropubic sling excision procedures, Fig. 4, 5 and 6 for examples of obturator sling excision procedures, and Figs. 7, 8 and 9 for examples of TVM mesh excision.

Note the following:

- These terms refer to surgical interventions; office-based mesh trimming procedures are not included.

- The aforementioned terminology should not be used in determining Current Procedural Terminology coding or other billing processes.

\section{Literature review and proposed treatment algorithms for the treatment of mesh complications}

\section{Methods}

To define the treatment algorithm, the writing group adhered to an iterative Delphi-type process that started with the premise that the approach to treatment of mesh complications should begin with stratification by device type (MUS), TVM, and sacrocolpopexy (SCP). After stratifying by type of mesh device, specific conditions were identified as starting points for key decisions. Treatment options represent decision nodes, and the outcomes of those procedures provided a springboard for the next round of conditions with their respective treatment nodes. After each round of algorithm development and discussion, the decision nodes were investigated using PubMed literature search of relevant diagnosis and treatment terms, and the available 


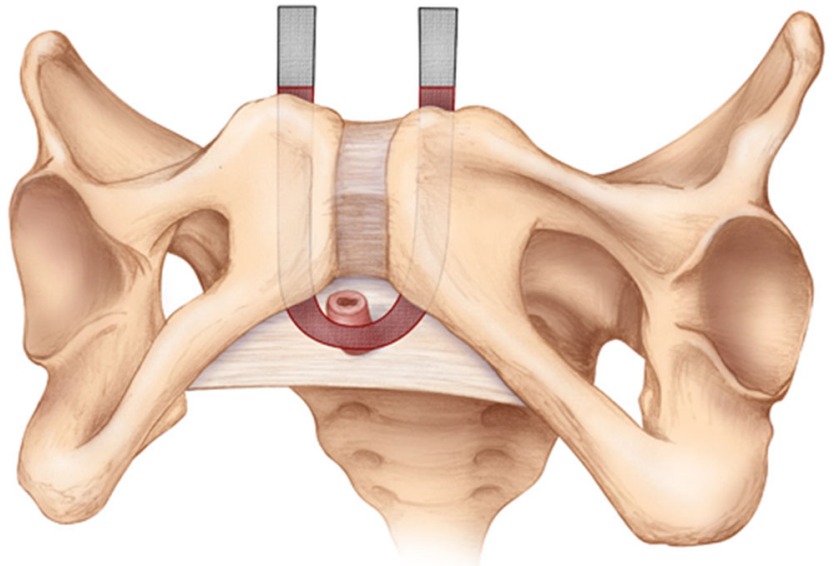

Fig. 1 Example with retropubic MUS: complete vaginal mesh excision and extravaginal mesh excision ("both retropubic arms"). Areas shaded red are excised. Note that this would not be considered total mesh excision because the suprafascial mesh is not removed in this example.

evidence was used to determine the level of support for each decision node, or when necessary, the algorithm was modified to reflect the relevant evidence. Each subsequent iteration of the algorithms involved a new literature review that was tailored to address questions and assess support for issues that arose with each iteration. The literature review for each node had a primary literature reviewer whose findings were assessed by a secondary review by another member of the Writing Group. No specific time points were excluded in the literature searches. The algorithms were further developed and refined based on these literature reviews, and the process was repeated until consensus was reached. The level of evidence supporting each node and the grade of corresponding recommendations were defined using an adaptation from the Canadian Task Force on Preventive Health Care for specific clinical preventive actions [5]:

Grades for recommendations are as follows:

A: There is good evidence supporting the recommended action.

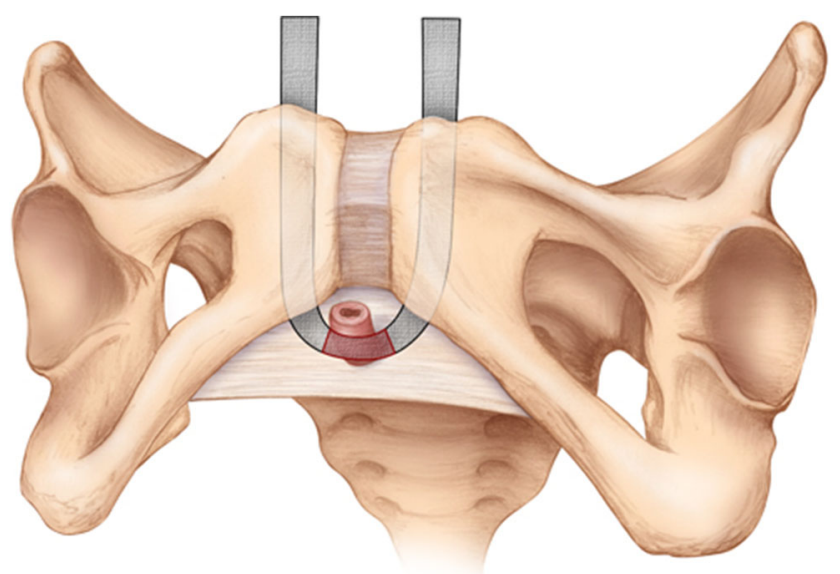

Fig. 2 Example with retropubic MUS: partial vaginal mesh excision. Area shaded red is excised.
B: There is fair evidence supporting the recommended action. $\mathrm{C}$ : There is conflicting evidence that limits the recommendation; other factors may influence decision making.

D: There is fair evidence recommending against the action.

$\mathrm{E}$ : There is good evidence recommending against the action.

I: There is insufficient evidence (in quantity, quality, or relevance) to make a recommendation; other factors may guide decision making.

The algorithms were formatted into a decision-making tool that is intended to be visually accessible, and to help patients and providers identify the relevant primary complication, explore treatment options, and understand the best available data supporting each option.

It is critical for a provider offering surgical treatment of mesh complications to understand that many patients will require multiple procedures for their complications, and the balance between undertreatment and overly aggressive treatment must be taken seriously. However, recognition that many patients require multiple surgical procedures to address mesh complications prompted this Writing Group to recommend that, in general, treatment options within each algorithm should not be repeated. For example, repetitive mesh revision procedures will complicate, or even render impossible, complete excision of the mesh. Therefore, if a given treatment is unsuccessful, consideration should be given to advancing to the next item on the algorithm to minimize the likelihood of multiple surgical events without clinical progress.

Special notes about the treatment algorithms are as follows:

- The algorithms in this Position Statement are not intended for use in the perioperative period. Immediate postoperative pain, neurologic complaints or findings, or other functional issues may have an immediacy and acuity that require swift diagnosis and treatment not relevant to the literature searched performed.

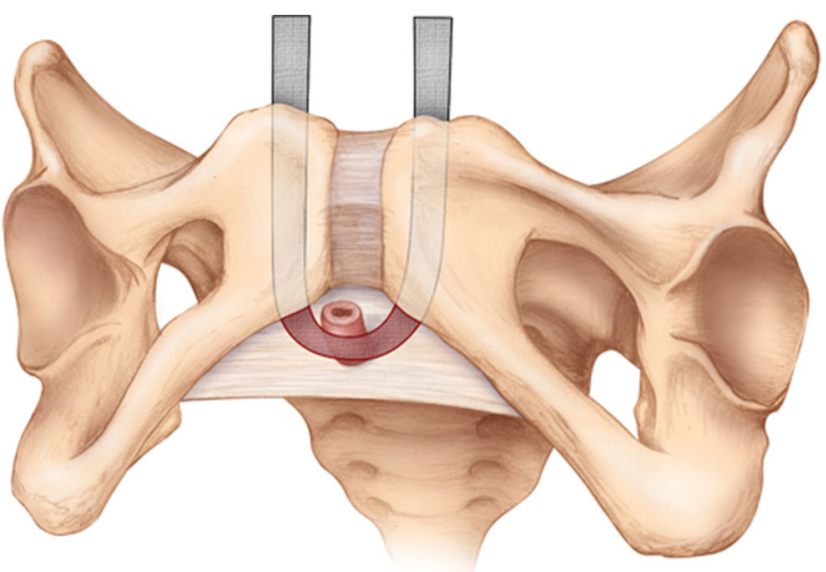

Fig. 3 Example with retropubic MUS: complete vaginal mesh excision. Area shaded red is excised. 


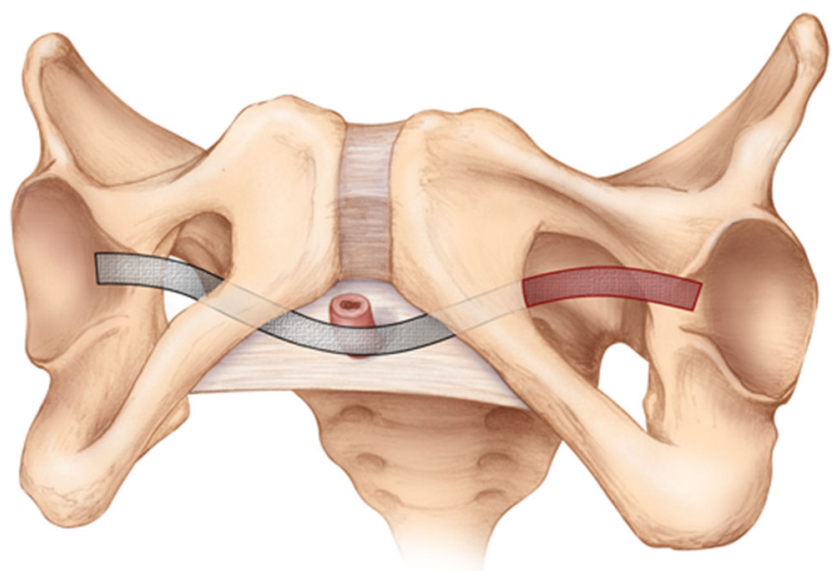

Fig. 4 Example with transobturator MUS: extravaginal mesh excision ("left groin sling arm"). Area shaded red is excised.

- These algorithms are applicable to patients with Amid type I, polypropylene mesh implants only. Host responses to implantation of other synthetic or biologic materials are too varied to consider in these algorithms.

- Because patients can be treated with more than one mesh implant, it is the responsibility of the provider, using appropriate diagnostic measures, to determine which device, and therefore which algorithm, is most salient for each patient.

- In instances when a patient has both mesh exposure and pain, management should be determined on the basis of pain. The coexistence of mesh exposure in the presence of pain seems to have little impact on the effectiveness of surgical treatment of pain [6].

\section{Mesh exposure of MUS}

Patients with MUS mesh exposures (Fig. 10, Table 2) who are asymptomatic and, in accordance with the National Institute

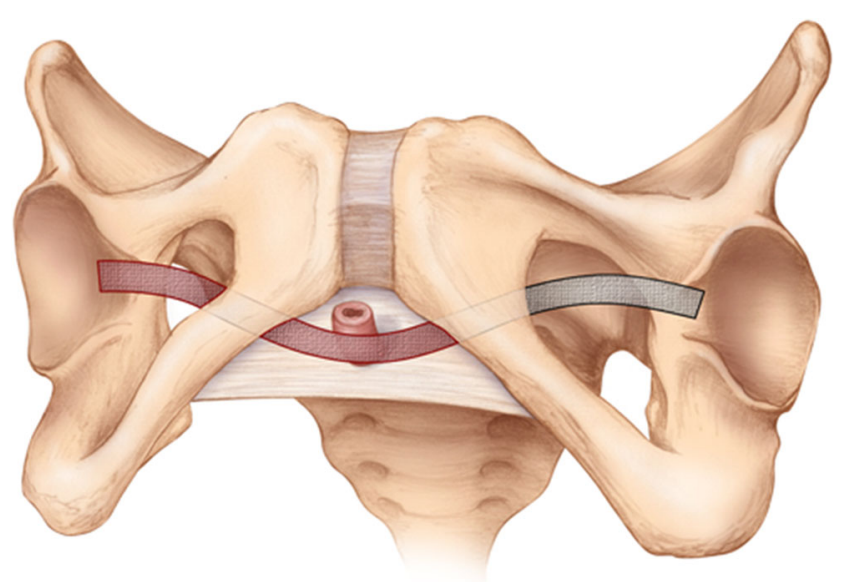

Fig. 5 Example with transobturator MUS: complete vaginal mesh excision and extravaginal mesh excision ("right groin sling arm"). Areas shaded red are excised.

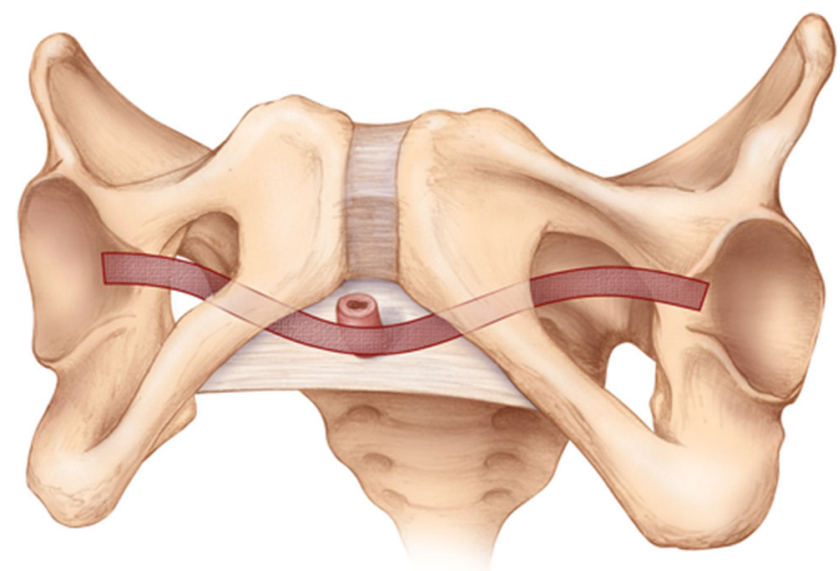

Fig. 6 Example with transobturator MUS: total mesh excision. Areas shaded red are excised.

for Health and Care Excellence document, have exposures smaller than $1 \mathrm{~cm}$ in size can often be safely observed. A small case series describes safe observation of asymptomatic MUS exposures with resolution [7], and type I polypropylene mesh exposures have been successfully observed in other settings [8]. However, after counseling, women without symptoms may still opt to proceed with excisional treatment. The value of adding topical estrogen is at best unclear.

Grade of recommendation: $\mathrm{C}$

MUS patients with symptoms from mesh exposure (eg, bleeding, discharge, and discomfort or partner pain with intercourse) may elect office-based trimming. However, the likelihood of success with this measure is not well described in MUS patients, and patients should be made aware of significant risk of recurrent/persistent mesh exposure [9]; one series showed that the three-quarters of women undergoing officebased trimming wound up with a surgical interventions (although MUS was not separated from other types of mesh)

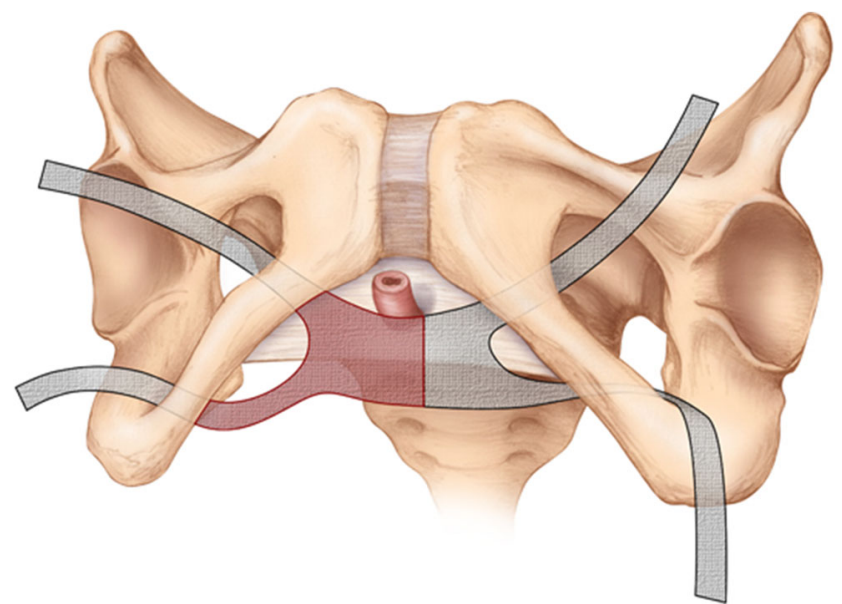

Fig. 7 Example with TVM: partial vaginal mesh excision and extravaginal mesh excision ("right mesh arms to the arcus tendinous and the sacrospinous ligament but not beyond"). Area shaded red is excised. 


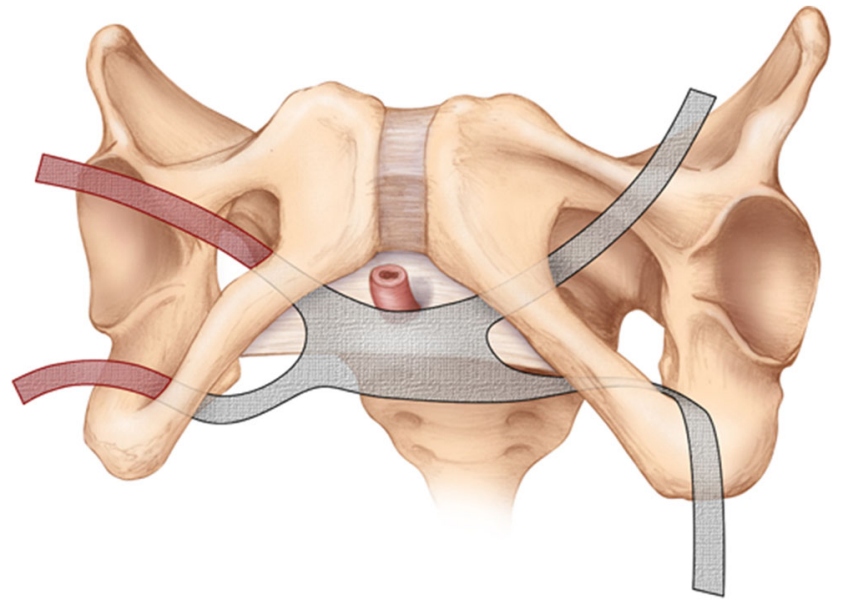

Fig. 8 Example with TVM: Extravaginal mesh excision ("both right groin arms"). Areas shaded red are excised.

[10]. Some published data on trimming or revising MUS mesh refer to "outpatient treatment" and do not describe anesthesia, the amount of mesh removed, or details on the setting of these procedures [11].

Grade of recommendation: C

For patients with bothersome MUS mesh exposure, surgical intervention should be considered, has high rates of resolution, and is the preferred approach in women for whom an office-based procedure has failed [11-13]. Described techniques include reclosure of the vaginal epithelium over exposed mesh (which would be classified as "mesh revision" in this document), as well as partial and complete vaginal mesh excision [14]. Although there is little guidance to determine the optimal amount of mesh to resect, the risk of persistent mesh exposure must be balanced with the increasing risk of SUI, as more mesh is resected; among women with reoperation for MUS sling exposure, partial excision resulted in $7 \%$ recurrent SUI, whereas complete vaginal excision resulted in 59\% recurrent SUI [15]. Assuming that there were no

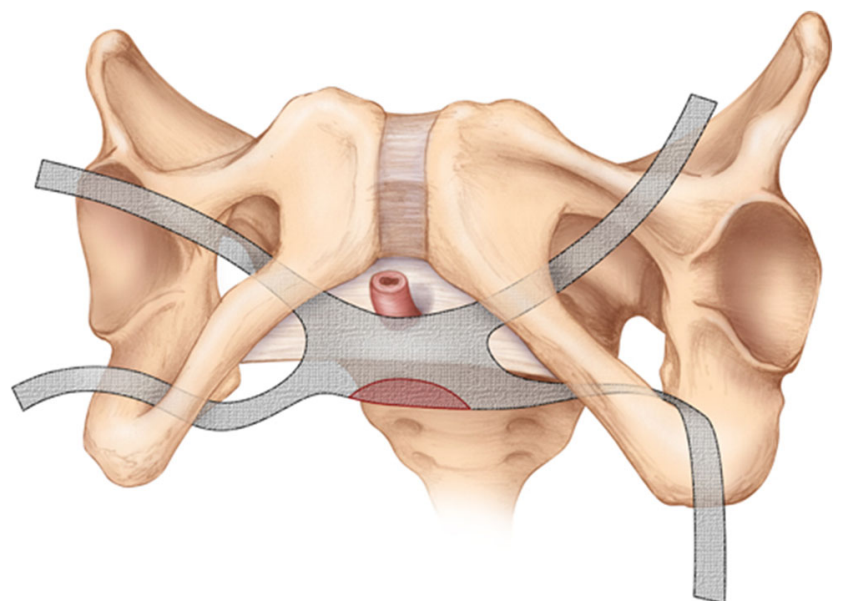

Fig. 9 Example with TVM: Mesh revision. Area shaded red is excised. Note that the mesh implant remains contiguous. recurrences of exposure, this evidence would favor partial vaginal mesh excision in the setting of vaginal exposures.

Grade of recommendation: B

Women with MUS mesh discovered in other organs, such as the bladder or urethra, can consider endoscopic approaches to removal of exposed portions. Urethral mesh exposures, for example, may be more amenable to transurethral or urethroscopic mesh excision than exposures higher in the urinary tract [16-19]. One study of transvaginal removal of urethral mesh exposures showed high rates of subsequent incontinence $(95 \%)$ but no additional treatments required for persistent mesh [20]. Although suture and mesh exposure in the bladder have been addressed with cystoscopic procedures including laser, when mesh is left in or near the initial point of exposure, recurrence rates are significant $[11,21]$. Generally, minimally invasive procedures introduce less morbidity [22], but they are associated with higher rates of persistence or recurrence of mesh exposure. Women may elect to proceed directly to more advanced mesh removal procedures if definitive resolution of the mesh exposure is their priority. The risk of recurrent SUI should be considered, but expert opinion suggests that implanting synthetic materials should be avoided at the time of urinary tract injury repair; some have advocated concurrent autologous sling to address this issue [23]. It is incumbent on the treating surgeon to have a thorough understanding of all organ systems potentially involved and to arrange for the appropriate consultative assistance before initiating surgical treatment.

Grade of recommendation: $\mathrm{C}$

\section{Pain associated with MUS}

Women with pain who are suspected of having a fibromuscular or myofascial component on examination may be candidates for referral to a physical therapist (PT) with expertise in treatment of pelvic floor dysfunction [24]. Midurethral slings - notably the full-length obturator-type slings - have been associated with pain in the groin $[25,26]$. Previous guidance documents recommend physical therapy (PT) for patients with pain at 6 weeks or more beyond implantation [1]. However, the evidence supporting the role of PT specifically for MUS-related pain is sparse and limited to case report [27, 28], and it is important not to delay other treatments if the pain does not respond to PT treatment (Fig. 11, Table 2).

Grade of recommendation: $\mathrm{C}$

Like PT, analgesic and/or steroid injections can have both diagnostic and therapeutic benefits [29], but their role has not been well defined with regard to MUS complications. Alternative treatments, including surgery, should be considered if repetitive injections or other interventional therapies become necessary.

Patients with pain that is attributable to the presence of MUS may be candidates for surgical excision in the form of 

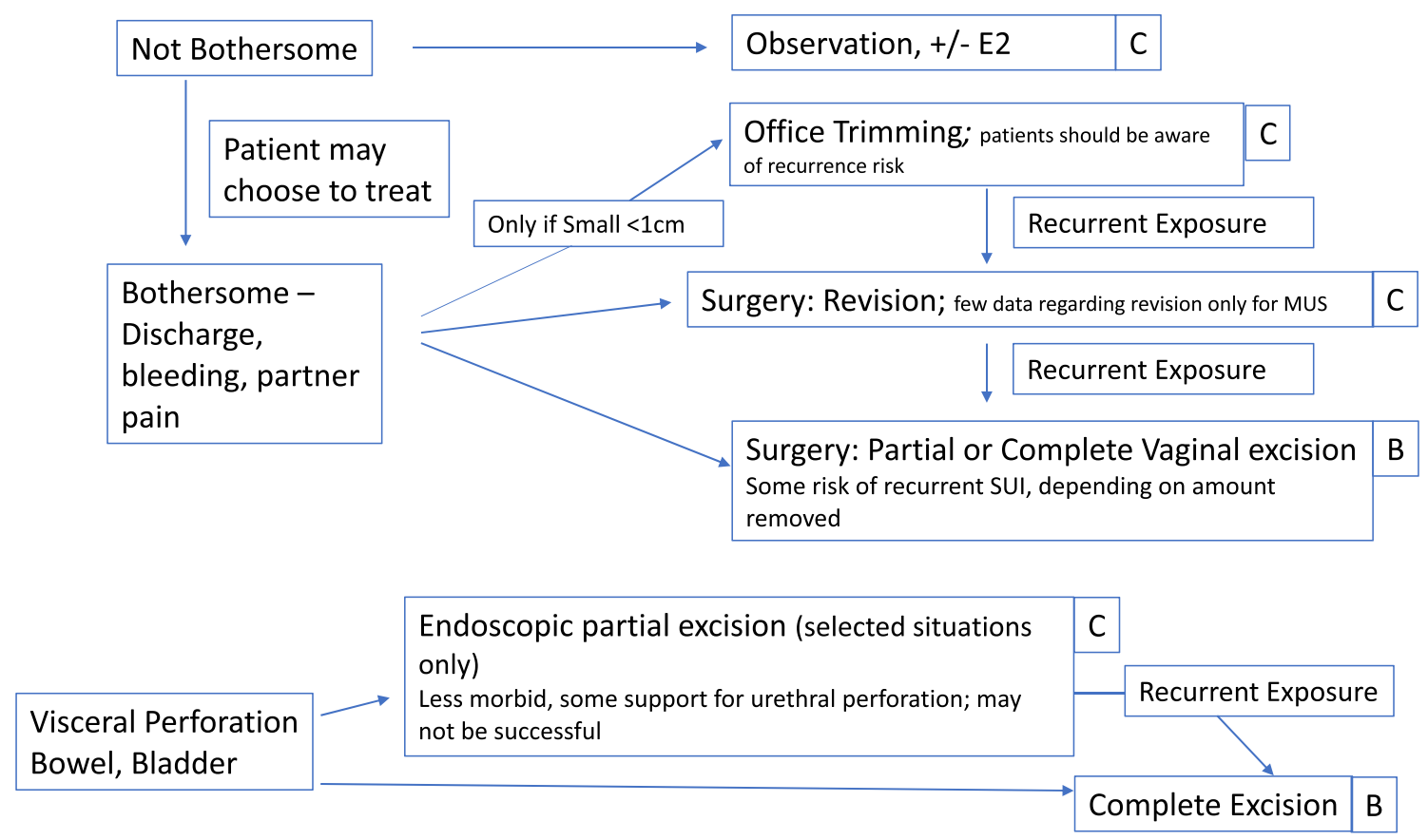

Fig. 10 MUS: exposure, no pain. Letters refer to grade of recommendation (Table 2).

partial or complete vaginal mesh excision. However, there are conflicting data concerning pain resolution in relation to the amount of mesh that is removed. Some case series show no differences in pain resolution after partial versus complete resection, whereas others describe patients who required additional procedures because of unsuccessful partial excisions. Offsetting the risk associated with incomplete treatment with partial excision is the increased risk of surgical complications or recurrent SUI that is associated with more extensive resection $[26,30]$. Again, there is little guidance to determine the amount of mesh to be excised; among patients with reoperation for MUS-related pain, partial versus complete vaginal excision yielded similar rates of pain resolution $(72 \%-76 \%)$ and the rates of SUI ( $22 \%$ for partial, $56 \%$ for complete) were not statistically significantly different [15]. It is important for the patient to realize that most data suggest that some degree of pain remains for onequarter to one-half of patients [30-32].

Grade of recommendation: B

Table 2 Mesh Complication Treatment Algorithms*

- Intended to apply to type I polypropylene mesh only $\circ$ Characteristics and risks of infection are likely different with other mesh or biologic materials.

- In general, repeating the same steps in the treatment algorithm is discouraged.

- Repetitive trimming may make complete removal difficult or impossible.

-When pain and mesh exposure coexist, pain should be the driving consideration.

- Not all possible courses of action are included. $\circ$ Actions are only included if there is reasonable evidence support.

*See Figs. 10, 11, 12, 14, and 15.
Some patients may experience pain related to MUS that is not directly linked to the vaginal portion of the sling. Pain related to a retropubic MUS that is elicited with retropubic palpation, or pain that is refractory to more conservative measures, can be addressed with removal of the retropubic portions of the mesh with good results [33, 34]. Favorable results with both laparoscopic and open approaches have been described.

Grade of recommendation: B

For patients with transobturator MUS-related groin pain, release of tension by surgical removal of the vaginal portion of the sling is successful in most cases [30,35]. Surgical exploration of the groin is usually not required and should be limited to cases that have not responded to vaginal excision, or where pain is elicited in the groin on examination [26].

Grade of recommendation: $\mathrm{C}$

Extravaginal excision of the groin and/or thigh compartment segments of mesh, should be reserved for cases that are refractory to more conservative measures, and/or when there is evidence of nerve impingement (with discrete and dermatomal distribution consistent with anatomic location) or if there is evidence of suppurative infection. One report described 22 patients with transobturator tape-related vaginal and groin pain who underwent both vaginal and groin/thigh mesh resection, but did not report outcomes for these interventions [33]. Another report described removal of a portion of the groin/thigh part of mesh from 2 patients, but both had severe and immediate pain accompanied by considerable incapacitation [36]. Although these dramatic, short-term surgical outcomes are beyond the scope of this document, at this time, we believe that extravaginal mesh excision from the 

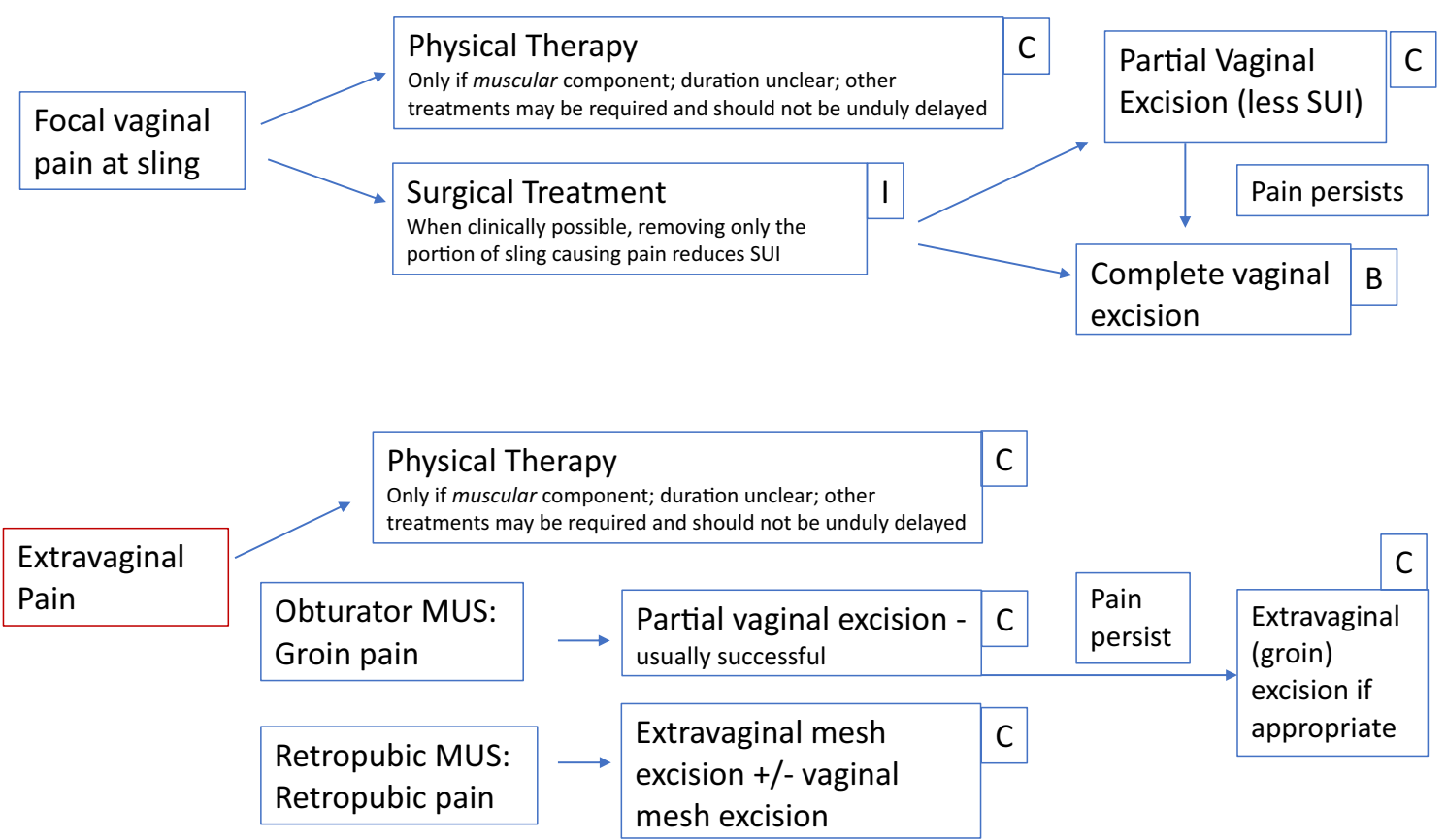

Fig. 11 MUS: pain/dyspareunia attributed to sling. Letters refer to grade of recommendation (Table 2).

groin is rarely indicated and should only be performed by a surgical team with appropriate multidisciplinary expertise.

Grade of recommendation: $\mathrm{C}$

\section{Exposure of SCP mesh}

Although there are limited data describing this scenario, a case series of small and asymptomatic TVM mesh exposures showed no symptom development over an average of 10 years of follow-up, and it may be reasonable to extrapolate these findings to SCP when similar materials are used [8]. The exposure is unlikely to resolve with observation with or without topical estrogen; one series showed that only one-quarter of SCP mesh exposures resolved spontaneously or with topical estrogen [37]. There is some concern about ascending infection from mesh exposure that may lead to osteitis of the

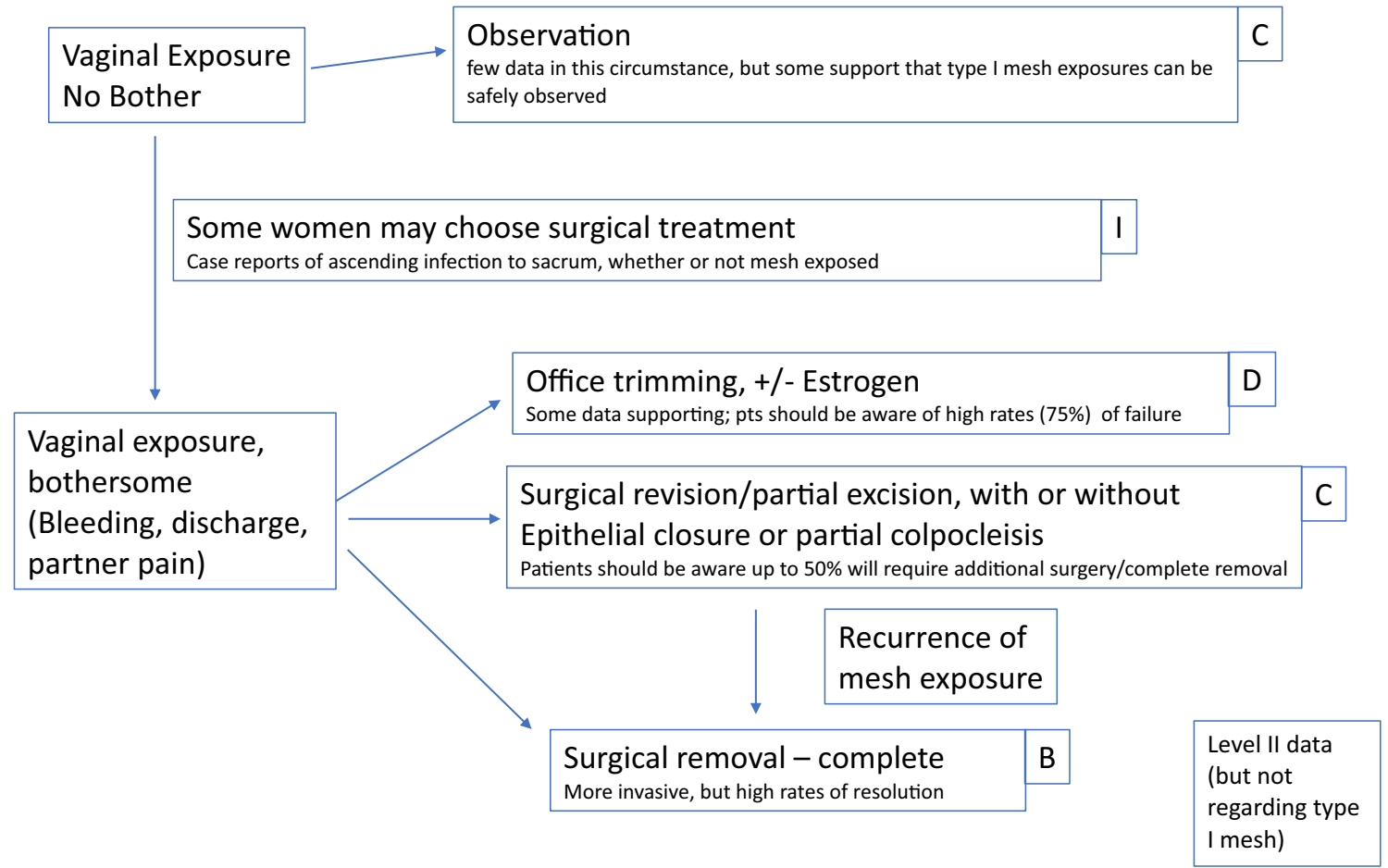

Fig. 12 Sacrocolpopexy: mesh exposure (without pain). Letters refer to grade of recommendation (Table 2). 
sacrum, particularly among patients with earlier generation mesh implants $[38,39]$. On the other hand, there are also reports of similar ascending infections among women without mesh exposure [40]. In the absence of clear evidence of infection or sinus tract formation, surgical intervention for asymptomatic mesh exposure is likely not required, although patients may still elect to have it removed (Fig. 12, Table 2).

Grade of recommendation: $\mathrm{C}$

For patients with symptomatic mesh exposures (eg, bleeding, discharge, or partner pain), office-based trimming can be considered. However, current literature suggests that most patients will ultimately require surgical intervention. One report of 19 women with mesh exposure after apical mesh repair showed that only one-quarter resolved with observation with or without topical estrogen, and that office-based trimming was ineffective [37]. These patients may therefore elect to decline a potentially uncomfortable office procedure and to proceed to surgical intervention.

Grade of recommendation: D

Repetitive trimming procedures are not recommended. Expert opinion dictates that repeated disruption of the integrity of the mesh may make it more difficult to remove the mesh in its entirety should it become necessary.

For patients who elect surgical revision due to mesh exposure, partial mesh excision, with epithelial closure and/or partial colpocleisis, can be considered; 2 series, each with 5 or fewer patients with SCP mesh exposures, were successfully managed with vaginal excision only [41, 42]. However, patients should be counseled about the likelihood of eventually requiring complete surgical excision. Several series of mesh excision procedures for exposure demonstrate that $37 \%$ to $50 \%$ of patients undergoing vaginal mesh excision went on to require additional procedures [43-46]. Of note, Quiroz et al $[18,44]$ found that none of the second (or third) vaginal mesh excision procedures were successful, and total mesh removal was required in all cases after failed vaginal mesh resection. Although these studies include data from patients with heavier-weight polypropylene devices, or other materials including polyester, and thus, their relevance to lightweight polypropylene mesh is unclear, it illustrates the recommendation that procedures on the algorithms not be repeated.

Grade of recommendation: $\mathrm{C}$

\section{Pain associated with SCP mesh}

Pain and mesh exposure can overlap in patients with a history of SCP, (Fig. 13, Table 2) and in these patients, more is known about the outcomes of surgical intervention in terms of its impact on mesh exposure than on pain. One report that included 11 women with SCP mesh-related pain demonstrated that pain resolved in all women after surgical removal of some or all of the mesh; this study was unable to comment on postexcision dyspareunia [9]. More than one-half of these patients were successfully treated with vaginal mesh revision alone, although many also required abdominal surgery, and $25 \%$ required more than one surgery. However, details of these patients and the procedures they underwent were not described. Patients with pain were more likely to require total mesh excision than patients with vaginal mesh exposure only [41]. One series of 3 patients with persistent apical pain after SCP and without vaginal mesh exposure responded favorably to complete abdominal mesh resection; it should be noted that 2 of these 3 were also found to have suture material in the bladder, again illustrating the need for thorough preoperative evaluation [47].

Grade of recommendation: $\mathrm{C}$

In some cases, pain associated with SCP mesh may not be elicited with palpation of focal areas of the mesh during examination. Attribution of the pain to the mesh should be made with caution in these circumstances. However, if nonsurgical interventions have been exhausted and the patient elects to undergo mesh removal to address her pain, removal of the entire mesh should be given some consideration so that the patient is not subjected to multiple procedures.

Grade of recommendation: I

\section{Infection associated with SCP mesh}

Type I polypropylene mesh is generally considered more resistant to infection than many antecedent mesh materials. Although the incidence of infections that are attributable to this mesh is low, guidance for treatment of these infections is also lacking. One report described 22 cases of infected mesh, but most involved grafts were not polypropylene, and none were lightweight, type I mesh. However, all cases involved mesh exposure, and all were successfully treated with surgical excision. A case report describing a sinus tract formation around a non-type I mesh SCP that progressed to sacral abscess also provides indirect support for surgical excision of infected mesh [39].

Grade of recommendation: B

Data from a series of 3 patients suggest that conservative management of pelvic abscesses after SCP procedures should be considered [48]. Although none of the patients in this report had exposed mesh, all infections occurred within 14 days of SCP surgery. In these 3 cases, conservative management with computed tomography-guided drainage and antibiotics was successful.

Grade of recommendation: $\mathrm{C}$

In cases of persistent pain attributable to the mesh after partial mesh excision procedures, complete mesh removal is recommended. One series demonstrated that up to half of patients with pain related to silicone mesh were inadequately treated with a vaginal approach and went on to require complete/abdominal mesh excision (again, relevance to type I mesh is unclear) [49]. As noted previously, the current 

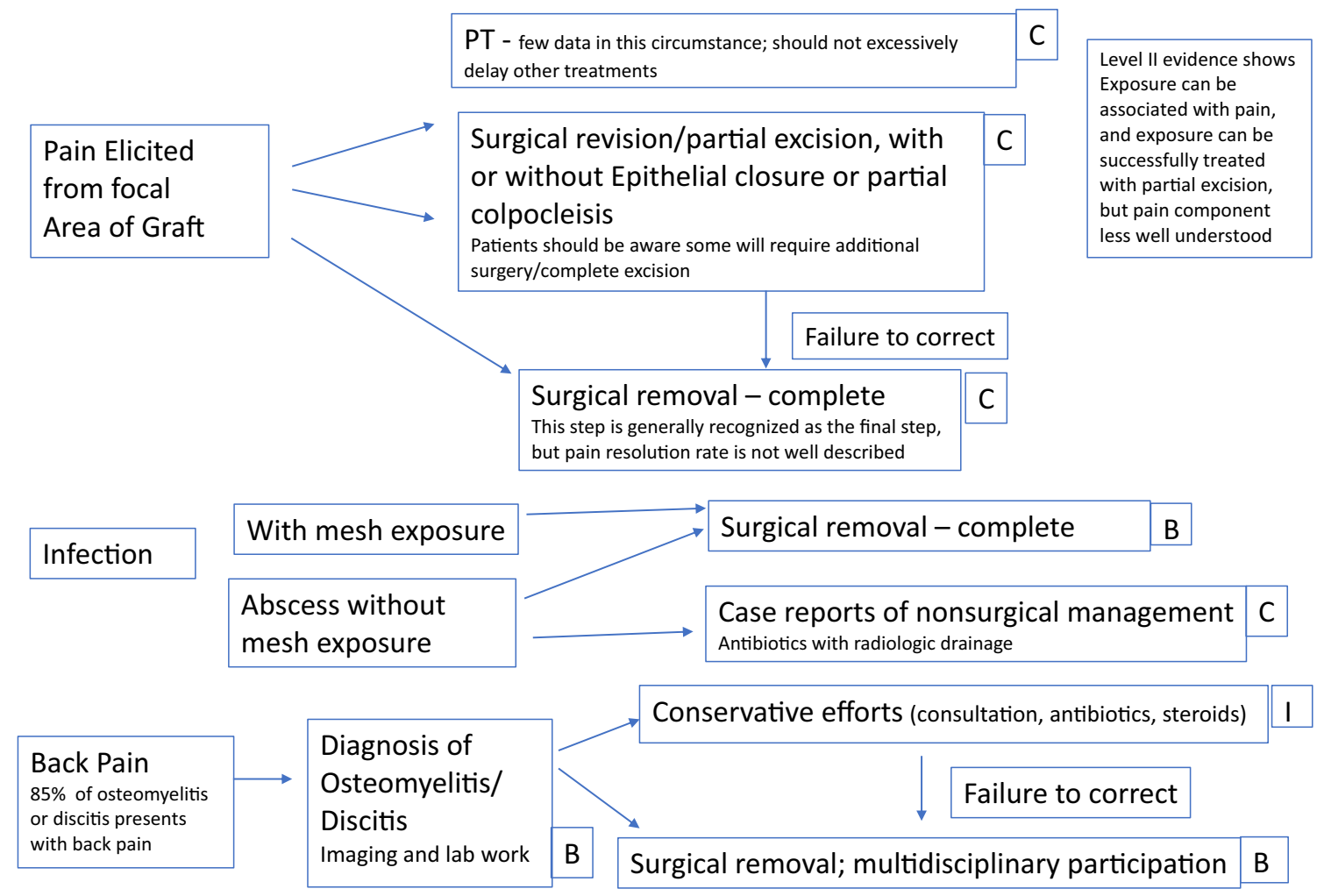

Fig. 13 Sacrocolpopexy: pain or dyspareunia (with or without mesh exposure). Letters refer to grade of recommendation (Table 2).

available data are primarily focused on mesh exposure, some of which may be associated with pain, but the ability to comment on pain and the success of intervention is limited. However, patients should be aware that a significant number of patients treated with partial or complete vaginal mesh excision procedures will go on to require additional surgery for the removal of the abdominal portion.

Grade of recommendation: B

Evidence concerning the risk of recurrent prolapse after SCP mesh removal is sparse. One study of 22 women [46] reported an $18 \%$ recurrence, but the follow-up period was not specified. One study of 19 patients with pain and/or mesh exposure treated with total mesh excision (the outcomes for pain were not described) also received a concomitant SCP using rectus fascia; at 9-month follow-up, no recurrent prolapse was observed [50].

\section{Back pain in the setting of SCP}

Osteomyelitis or discitis is a rare but serious condition, and scientific evidence is limited to case report literature. However, all case reports were systematically reviewed by Muller et al. [51] Back pain is the most common symptom of sacral spondylodiscitis/osteomyelitis, present in $85 \%$ of cases, and therefore, persistent back pain should prompt a workup. The most common imaging study is magnetic resonance imaging; relevant serum markers of inflammation include white blood count, erythrocyte sedimentation rate, and C-reactive protein. Direct aspiration is the preferred method for culture, especially if blood cultures are negative. Some cases will respond to antibiotic therapy alone, although most reported cases progressed to surgical intervention.

Grade of recommendation: B

There is currently insufficient evidence to develop guidance about situations in which conservative efforts versus surgical removal are preferable. When undertaking conservative approaches, one should be ready to proceed with surgical intervention if the patient does not respond satisfactorily.

\section{Exposure of TVM for prolapse}

Patients with asymptomatic exposure of Type I mesh may be observed, with or without topical estrogen treatment. A report of 9 women with asymptomatic Type I mesh exposure were followed for an average of 10 years with no progression or development of symptoms [8]. However, asymptomatic patients may still elect to proceed with surgical intervention (Fig. 14, Table 2).

Grade of recommendation: $\mathrm{C}$

Patients with symptomatic mesh exposures (bleeding, discharge, discomfort, or partner pain) should be counseled that observation with or without topical estrogen is unlikely to be 
successful [12]. The 2019 National Institute for Health and Care Excellence report recommended that observation with estrogen treatment should be reserved for patients with small $(<1 \mathrm{~cm})$ single exposures, and that this treatment should be given for no more than 3 months before considering surgical intervention [52].

Grade of recommendation: B

The success rate for office-based mesh trimming is poorly described. One report showed that $73 \%$ patients undergoing surgical revision of TVM ( \pm MUS) mesh reported previously having office-based trimming, which was ultimately unsuccessful [10]. Another report that included 63 women with vaginal mesh exposures concluded that office-based mesh trimming was successful in resolving the issue less than half of the time [37]. Patients should be counseled that officebased trimming is associated with a high likelihood of failure, and the option of definitive surgical management should be discussed.

Grade of recommendation: D

For patients with mesh exposure who elect surgical treatment, or for whom office-based treatments were unsuccessful, surgical treatment involving removal of some or all of the vaginal mesh is recommended.

Grade of recommendation: B

The few studies specifically addressing nonpainful vaginal mesh exposure do not suggest an advantage of complete mesh excision over partial mesh excision, but do indicate that complication rates are higher with more aggressive resection [12, 53-55]. Therefore, among women with mesh exposure and no pain, partial mesh excision is recommended. Complete mesh excision may be considered for the relatively few recurrences. There is no evidence concerning how much mesh should be removed. Expert opinion dictates resecting all exposed meshes and dissecting until complete healthy tissue ingrowth is encountered.

Grade of recommendation: $\mathrm{C}$

Although there are some reports describing use of biologic grafts to interpose over the gaps where mesh excision has rendered primary closure of the epithelium impossible, there is insufficient comparative evidence to recommend this approach over healing by secondary intent.

\section{Pain related to TVM for prolapse}

Any woman experiencing pain who is suspected of having a fibromuscular or myofascial component on examination may be a candidate for referral to a physical therapist with expertise in treatment of pelvic floor dysfunction [56]. It is plausible that passage of mesh through or connection of mesh to pelvic musculature may be involved in the development of myofascial pain; other guidance documents recommend PT for patients experiencing pain at 6 weeks or more after implantation. It is important to consider that patients with meshrelated pain may also have conditions in which PT has been demonstrated to be effective. However, there is limited evidence concerning this treatment in cases of TVM-related pain, and it is important not to delay other treatments if pain advances or becomes intractable (Fig. 15, Table 2).

Grade of recommendation: I

Similarly, there may be diagnostic or therapeutic benefits to trigger point injections with steroid and/or analgesic medications in some patients. However, there is no evidence to support a recommendation to use this approach in all cases of TVM-related pain, and alternative treatments, including surgery, should be considered if repetitive injections become necessary.
Fig. 14 TVM: exposure (without pain). Letters refer to grade of recommendation (Table 2).

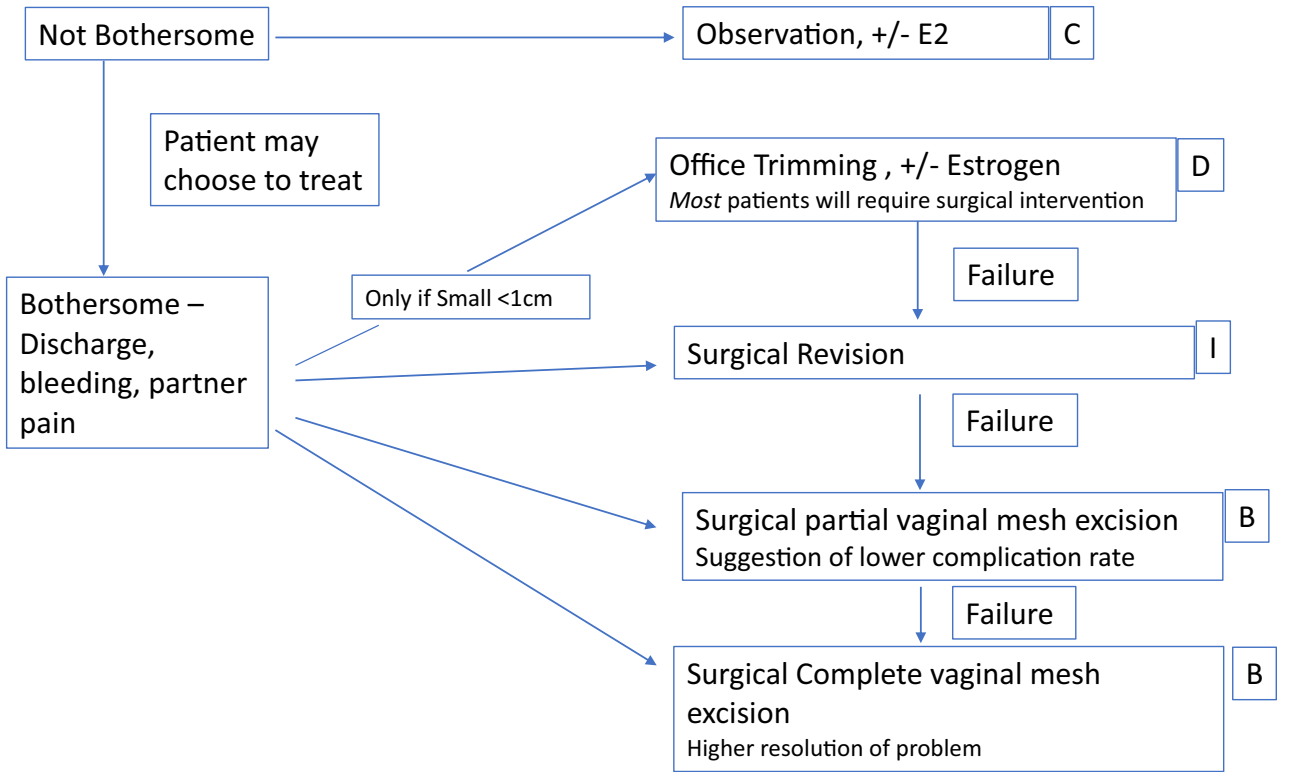


For patients with TVM-related pain that is refractory to conservative management, timely consideration of surgical treatment is recommended. There are no data supporting office-based revision procedures in this setting because the pain often relates at least in part to mechanical tension. Pain is more likely to be elicited over the attaching arms than central [57], and the goal of surgery should be either partial or complete vaginal excision, to allow for release of tension [58, 59]. Extravaginal mesh excision (eg, mesh arms of trocar-based TVM devices) is usually not required [60]. There are limited data concerning the decision to pursue partial or complete excision; one series found that in the anterior compartment, pain mapping was helpful in determining a surgical strategy [60], whereas another cited that in cases of pain as the presenting symptom, the entire portion of the mesh in the affected area was excised [61]. One study showed that health-related quality of life showed better response to complete vaginal mesh excision than to partial vaginal mesh excision [62]. The absence of randomized data further limits the ability to determine whether the incremental benefit of a complete vaginal mesh excision outweighs the additional risk. Whether a partial or complete mesh excision is planned, patients should be counseled that rates of some degree of residual pain been estimated at between $18 \%$ and $50 \%[12,55,62-65]$. One series showed no improvement in pain after partial or complete mesh excision in $16 \%$ [32]. Adjuvant therapies including pelvic PT should be used as appropriate.

Grade of recommendation: $\mathrm{C}$

\section{Patients with extrapelvic pain from TVM}

Some patients report pain that may be attributable to nerve impingement from mesh arms that are outside the pelvis (in the groin or in the ischiorectal fossa). In these cases, complaints should map to dermatomal distributions, and appropriate neurologic and radiologic evaluations should be carried out [66]. Anecdotal evidence has described extensive extravaginal mesh excision, with or without nerve release procedures; there are some data suggesting that this may be more successful in cases of obturator neuralgia than of pudendal neuralgia [35]. With regard to TVM-related conditions, outcomes of these procedures have been variable, and there is insufficient evidence to support this approach. Accordingly, extended extravaginal excision of TVM extrapelvic arms should be considered rarely.

Grade of recommendation: $\mathrm{C}$
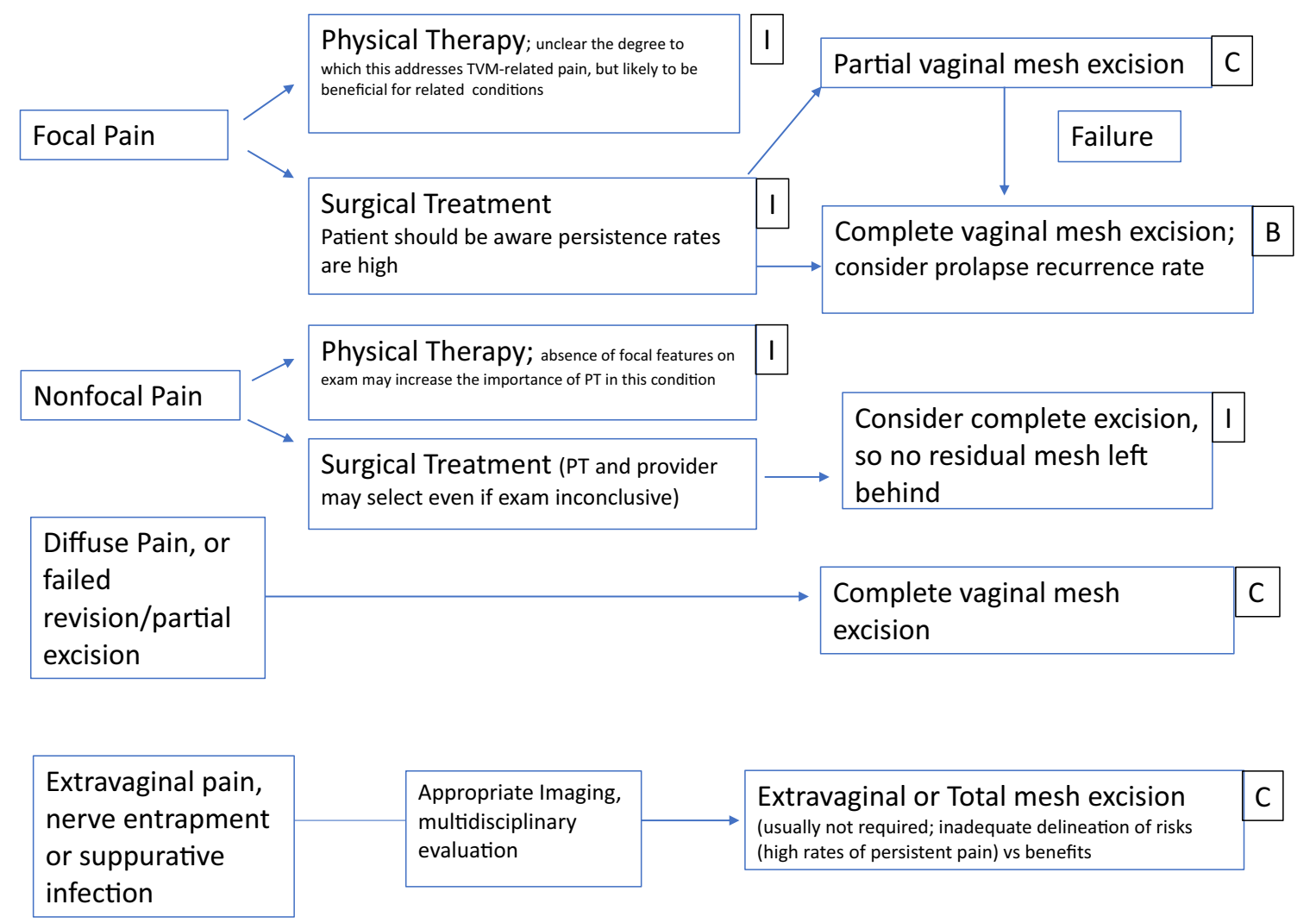

Fig. 15 TVM: pain or dyspareunia ( \pm exposure). Letters refer to grade of recommendation (Table 2). 


\section{Identification and prioritization of gaps}

Mesh complications - particularly those requiring surgical revision - are inherently highly individualized and sufficiently uncommon that randomized clinical trials aimed at identifying best practices are unlikely to be conducted in the near term. Given the dearth of experimental evidence, the grade of recommendation for our proposed algorithms varies between $\mathrm{B}$ and I. However, this does not eliminate the need for highquality data to drive informed decision making. It is therefore critical to shift our focus to a registry-based approach to evidence generation. If we can drive consistency in our terminology (both for the presenting condition and for its treatment) and encourage data capture from most, or all mesh complication cases, we will be better positioned to identify the best treatment options.

The value of better understanding of treatment options depends in part on the prevalence of mesh complications, which in turn depends on the frequency that specific devices or techniques are used. Evolving practice patterns and regulatory changes (including the Food and Drug Administration decision not to approve the TVM devices available at the time) have contributed to secular trends that not only changed the rate of TVM implantation but also created challenges in quantifying links between exposures and outcomes. Conversely, MUSs continue to be considered a standard of care for treatment of SUI, and the uptake of robotic surgery has increased the number of SCP procedures that are being performed. A key finding in this review is that much of the literature guiding treatment of SCP mesh complications is based on patients who were implanted with materials that are rarely used in current practice. We now understand that mesh characteristics such as filament type, density, porosity, and surface area significantly impact the behavior of the mesh in vivo, so we should be cautious about generalizing mesh complication management data that are based on patients whose implants contain materials that are no longer used. For this reason, as well as the increasing utilization of SCP in the era of robotic surgery, research on management of complications from type I polypropylene mesh exposure is an area of pressing need.

The following specific gaps in the evidence were identified:

- Success rates of partial mesh excision of exposed SCP mesh with lightweight monofilament mesh devices

- Safety of observation of asymptomatic mesh exposures in SCP

- Risk of prolapse recurrence following different types of SCP mesh excision

- Effects of surgical techniques on outcomes

- Specifics of surgical intervention for SCP-related pain
- Methods to determine whether to pursue partial or complete vaginal mesh excision in cases of TVM-related pain, and circumstances under which resection of the intrapelvic mesh arms is advisable

- Strategies to minimize postmesh resection prolapse

Research in these areas will make a meaningful contribution to informed decision making for patients and their care teams. The rate of TVM implantation has been steadily declining over the past few years, as fewer and fewer manufacturers have remained in the space; the recent decision by the Food and Drug Administration not to approve the 2 remaining TVM synthetic mesh devices available in the United States will very likely further reduce TVM implant rates to an uncommon and highly individualized procedure. Meanwhile, the adoption of minimally invasive and robotic surgical approaches has made the SCP a more popular procedure. Therefore, it seems prudent to turn our research attentions to complications related to MUS and SCP.

\section{Characteristics of providers and treatment centers that may optimize outcomes: exploratory and aspirational goals}

Debate still lingers in some areas regarding the role of subspecialty training and certification in the treatment for women with pelvic floor disorders in general, and in the management of mesh-related complications in particular. Although the American College of Obstetricians and Gynecologists/AUGS Committee Opinion describes recommendations for the $\mathrm{OB} /$ Gyn, the core curriculum in OB/Gyn training [67], as described in the CREOG Educational Objectives, does not specifically mention pelvic floor surgery complications or mesh-related complications [68]. Conversely, the ABOG Guide to Learning, which serves as the core curriculum for FPMRS training in the United States, includes diagnosis and management of meshrelated complications. For this reason, we believe that meshrelated complications are best managed by FPMRS subspecialists or, in countries without subspecialty certification, by those whose practice is primarily dedicated to FPMRS conditions.

We believe that although mesh implant and mesh excision are undoubtedly related procedures, they differ in meaningful ways that require treating surgeons to reflect with great care on their training and experience. Historically, many surgeons have espoused the concept that mesh should only be implanted by providers who are skilled enough to take it out. This is supported by some older evidence from mesh referral centers that only $14 \%$ of patients were referred for treatment by the implanting surgeon [69]. However, this principle may not be in the patient's best interest because it might encourage implanting surgeons to undertake excision procedures that are 
beyond their comfort or skill level. Newer data suggest that there may be an increasing pattern of referral to subspecialty centers; compared with facilities that perform surgical prolapse repair alone, those where surgery for mesh complications is performed are fewer in number and are concentrated in metropolitan areas [70].

A favorable association between surgical volume, at both individual and institutional levels, and clinical outcomes has been demonstrated across multiple fields of surgery. However, the association between mesh complication surgery volume and outcomes is evolving because these procedures are relatively recent and reflect the rapidly evolving field of meshbased surgical technologies. As highlighted earlier in this document, most published literature that is available on these outcomes comes from a few high-volume centers or subspecialist practices. Although many of these case series suggest that mesh excision can be performed in a minimally invasive fashion with relatively low risk of perioperative complications $[8,9,13-19,21,22]$, others describe major perioperative complications $[10,12,20]$. These heterogenous results underscore the importance of the decision to pursue surgery, and they also suggest that referral to a provider/center with specific expertise in these procedures is prudent.

Management of mesh-related complications should center around the patient, who may elect to see her initial surgeon or elect to be referred elsewhere. In either case, optimal management of mesh complications may be achieved with a multidisciplinary team. The United Kingdom offers an example of this model, which is considered good practice for treatment of chronic conditions because it encourages appropriate, highquality care for all patients. Having access to a board of clinicians with different skills enables patients to access a breadth of different treatments rather than the one provided by a specific individual [71].

Ideally, the multidisciplinary team would also use standardized nomenclature for diagnosis and classification of mesh complications, and for treatment procedures, as well as participate in registries designed specifically for the treatment of mesh-related complications.

\section{Conclusions}

As providers of care for women with pelvic floor disorders, we recognize that any surgical intervention is associated with risks and that mesh implantation carries unique risks that are specific to the implant. By definition, providers offering mesh-based procedures interpret available data to mean that, on the whole, benefits of implanting mesh outweigh the risks. However, this may not be the experience of individual patients.

To date, our ability to synthesize robust, collective experience and expertise about management of mesh complications has been hampered by the rapid proliferation of devices and materials, each with different in vivo characteristics. These challenges have been exacerbated by our lack of adoption of consistent terminology, the impact of the relative infrequency of these complications on published data, and the absence of data systems to collect standardized data on these events. Against this backdrop, surgeons who perform mesh complication procedures have different experience levels and training backgrounds, and information derived from high-volume, highly trained surgeons may have limited applicability to the larger population of clinicians who care for patients with mesh complications.

AUGS and IUGA — societies whose primary missions are to improve care for women with pelvic floor disordersbelieve that women with mesh complications will be better served if the following are present:

- Consistent terminology for the diagnosis and description of mesh complications, as well as the procedures to treat them

- Improved ability to delineate and publicize centers with superior experience and services that are most likely to help a patient resolve a mesh complication

- The availability of treatment algorithms that are based on specific complications that can assist in shared decision making

- A shift in research resources to focus on the most important gaps in the literature concerning treatment of mesh complications

- Availability of comprehensive, high-quality registry data that inform on optimizing treatment of mesh complications in real time

Finally, it is our responsibility to provide evidence derived from scientific experience to fill the void that is, in some cases, allowing other forces to alarm or mislead patients.

Acknowledgments The authors wish to acknowledge the work of Cundiff, Quinlan, van Rensburg, and Slack, whose publication "Foundation for an evidence-informed algorithm for treating pelvic floor mesh complications: a review" was the formative starting point for the device-stratified management algorithm aspect of this project (Cundiff GW, Quinlan DJ, van Rensburg JA, et al. Foundation for an evidenceinformed algorithm for treating pelvic floor mesh complications: a review. BJOG 2018;125(8):1026-1037).

The following are being recognized for their contributions to this article: Charles R. Rardin, MD (writing group chair) (Division of Urogynecology and Reconstructive Pelvic Surgery, Department of Obstetrics and Gynecology, Alpert Medical School of Brown University, Providence, RI); Jonathan Duckett, MD (Department of Obstetrics and Gynaecology, Medway Maritime Hospital, Gillingham, Kent, United Kingdom); Alfredo L. Milani, MD (Department of Obstetrics and Gynecology, Reinier de Graaf Hospital, Delft, the Netherlands); Lucila Ines Paván, MD (Division of Urogynecology, Hospital Italiano de Buenos Aires, Buenos Aires, Argentina); Lisa Rogo-Gupta, MD (Departments of Gynecology and Urology, Stanford University, Stanford, CA); Christian O. Twiss, MD (Department of Urology, University of Arizona, Tucson, AZ). 


\section{Compliance with ethical standards}

Conflict of interest Dr Rardin receives research support from the Eunice Kennedy Shriver National Institute of Child Health and Human Development; the Foundation for Female Health Awareness, Pelvalon, Inc; and Solace Therapeutics. Dr Duckett received support to attend the International Urogynecological Association's annual meeting in Nashville from Contura. Drs Milani, Paván, Rogo-Gupta, and Twiss report no conflicts of interest.

\section{References}

1. Management of mesh and graft complications in gynecologic surgery. Committee Opinion No. 694. American College of Obstetricians and Gynecologists, American Urogynecologic Society. Female Pelvic Med Reconstr Surg. 2017;23(3):171-6.

2. Gyang AN, Feranec JB, Patel RC, et al. Managing chronic pelvic pain following reconstructive pelvic surgery with transvaginal mesh. Int Urogynecol J. 2014;25:313-8.

3. Crosby EC, Abernethy M, Berger MB, et al. Symptom resolution after operative management of complications from transvaginal mesh. Obstet Gynecol. 2014;123(1):134-9.

4. Haylen BT, Freeman RM, Swift SE, et al. International Urogynecological Association; International Continence Society; Joint IUGA/ICS Working Group on Complications Terminology. An International Urogynecological Association (IUGA)/ International Continence Society (ICS) joint terminology and classification of the complications related directly to the insertion of prostheses (meshes, implants, tapes) and grafts in female pelvic floor surgery. NeurourolUrodyn. 2011;30(1):2-12.

5. Canadian Task Force on Preventive Health Care. New grades for recommendations from the Canadian Task Force on Preventive Health Care. CMAJ. 2003;169:207-8.

6. Danford JM, Osborn DJ, Reynolds WS, et al. Postoperative pain outcomes after transvaginal mesh revision. Int Urogynecol J. 2015;26(1):65-9.

7. Kobashi KC, Govier FE. Management of vaginal erosion of polypropylene mesh slings. J Urol. 2003;169:2242-3.

8. Deffieux X, Thubert T, de Tayrac R, et al. Long-term follow-up of persistent vaginal polypropylene mesh exposure for transvaginally placed mesh procedures. Int Urogynecol J. 2012;23(10):1387-90.

9. Hammett J, Peters A, Trowbridge E, et al. Short-term surgical outcomes and characteristics of patients with mesh complications from pelvic organ prolapse and stress urinary incontinence surgery. Int Urogynecol J. 2014;25:465-70.

10. Abbott S, Unger CA, Evans JM, et al. Evaluation and management of complications from synthetic mesh after pelvic reconstructive surgery: a multicenter study. Am J Obstet Gynecol. 2014;210: 163.e1-8.

11. Padmanabhan P, Hutchinson RC, Reynolds WS, et al. Approach to management of iatrogenic foreign bodies of the lower urinary tract following reconstructive pelvic surgery. J Urol. 2012;187:1685-90.

12. Tijdink MM, Vierhout ME, Heesakkers JP, et al. Surgical management of mesh-related complications after prior pelvic floor reconstructive surgery with mesh. Int Urogynecol J. 2011;22:1395-404.

13. Fabian G, Kociszewski J, Kuszka A, et al. Vaginal excision of the sub-urethral sling: analysis of indications, safety and outcome. Arch Med Sci. 2015;11(5):982-8.

14. Kershaw V, Nicholson R, Ballard P, et al. Outcome of surgical management for midurethral sling complications: a multicentre retrospective cohort study [published online ahead of print January 7 , 2019]. Int Urogynecol J. doi: 10.1007/s00192-018-3853-6.
15. Jambusaria LH, Heft J, Stuart Reynolds W, et al. Incontinence rates after midurethral sling revision for vaginal exposure or pain. Am J Obstet Gynecol. 2016;215(6):764.e1-5.

16. Plowright LN, Duggal B, Aguilar VC, et al. Endoscopic transurethral resection of urethral mesh erosion with the use of a pediatric nasal speculum. Obstet Gynecol. 2013;121(Pt 2 Suppl 1):440-3.

17. Wijffels SA, Elzevier HW, Lycklama a Nijeholt AA. Transurethral mesh resection after urethral erosion of tension-free vaginal tape: report of three cases and review of literature. Int Urogynecol Pelvic Floor Dysfunct. 2009;20:261-3.

18. Quiroz LH, Cundiff GW. Transurethral resection of tension-free vaginal tape under tactile traction. Int Urogynecol J Pelvic Floor Dysfunct. 2009;20:873-5.

19. Velemir L, Amblard J, Jacquetin B, et al. Urethral erosion after suburethral synthetic slings: risk factors, diagnosis, and functional outcome after surgical management. Int Urogynecol J. 2008;19: 999-1006.

20. Kowalik CG, Cohn JA, Kakos A, et al. Road to recovery after transvaginal surgery for urethral mesh perforation: evaluation of outcomes and subsequent procedures. Int Urogynecol J. 2018;29(6):887-92.

21. Ogel CA, Linder BJ, Elliott DS. Holmium laser excision for urinary mesh erosion: a minimally invasive treatment with favorable longterm results. Int Urogynecol J. 2015;26:1645-8.

22. Kim JH, Doo SW, Yang WJ, et al. Laparoscopic transvesical excision and reconstruction in the management of mid-urethral tape mesh erosion and stones around the bladder neck: initial experiences. BJU Int. 2012;110:E1009-13.

23. Shah K, Nikolavsky D, Gilsdorf D, et al. Surgical management of lower urinary mesh perforation after mid-urethral polypropylene mesh sling: mesh excision, urinary tract reconstruction and concomitant pubovaginal sling with autologous rectus fascia. Int Urogynecol J. 2013;24:2111-7.

24. Talli Y, Rosenbaum PT, Owens A. The role of pelvic floor physical therapy in the treatment of pelvic and genital pain-related sexual dysfunction (CME). J Sex Med. 2008;5:513-23.

25. Scottish Independent Review of the use, safety and efficacy of transvaginal mesh implants in the treatment of stress urinary incontinence and pelvic organ prolapse in women. Available at: https:// www.gov.scot/publications/scottish-independent-review-usesafety-efficacy-transvaginal-mesh-implants-treatment9781786528711/. Accessed 2 Sept 2019.

26. Agnew G, Dwyer PL, Rosamilia A, et al. Functional outcomes following surgical management of pain, exposure or extrusion following a suburethral tape insertion for urinary stress incontinence. Int Urogynecol J. 2014;25(2):235-9.

27. Cadish LA, Hacker MR, Modest AM, et al. Characterization of pain after inside-out transobturator midurethral sling. Female Pelvic Med Reconstr Surg. 2014;20(2):99-103.

28. Parnell BA, Johnson EA, Zolnoun DA. Genitofemoral and perineal neuralgia after transobturator midurethral sling. Obstet Gynecol. 2012;119(2 Pt 2):428-31.

29. Duckett JR, Jain S. Groin pain after a tension-free vaginal tape or similar suburethral sling: management strategies. BJU Int. 2005;95(1):95-7.

30. Rigaud J, Pothin P, Labat JJ, et al. Functional results after tape removal for chronic pelvic pain following tension-free vaginal tape or transobturator tape. J Urol. 2010;184(2):610-5.

31. Ismail S, Chartier-Kastler E, Reus C, et al. Functional outcomes of synthetic tape and mesh revision surgeries: a monocentric experience. Int Urogynecol J. 2019;30:805-13.

32. Hou JC, Alhalabi F, Lemack GE, et al. Outcome of transvaginal mesh and tape removed for pain only. J Urol. 2014;192(3):856-60.

33. Misrai V, Rouprêt M, Xylinas E, et al. Surgical resection for suburethral sling complications after treatment for stress urinary incontinence. J Urol. 2009;181(5):2198-202. 
34. Miklos JR, Chinthakanan O, Moore RD, et al. Indications and complications associated with the removal of 506 pieces of vaginal mesh used in pelvic floor reconstruction: a multicenter study. Surg Technol Int. 2016;29:185-9.

35. Marcus-Braun N, Bourret A, von Theobald P. Persistent pelvic pain following transvaginal mesh surgery: a cause for mesh removal. Eur J Obstet Gynecol Reprod Biol. 2012;162:224-8.

36. Hazewinkel MH, Hinoul P, Roovers JP. Persistent groin pain following a trans-obturator sling procedure for stress urinary incontinence: a diagnostic and therapeutic challenge. Int Urogynecol $\mathbf{J}$ Pelvic Floor Dysfunct. 2009;20(3):363-5.

37. Wong KS, Nguyen JN, White T, et al. Adverse events associated with pelvic organ prolapse surgeries that use implants. Obstet Gynecol. 2013;122(6):1239-45.

38. Taylor GB, Moore RD, Miklos JR. Osteomyelitis secondary to sacral colpopexy mesh erosion requiring laminectomy. Obstet Gynecol. 2006;107(Pt 2):475-7.

39. Hart SB, Weiser EB. Abdominal sacral colpopexy mesh erosion resulting in a sinus tract formation and sacral abscess. Obstet Gynecol. 2004;103:1037-40.

40. Grimes CL, Tan-Kim J, Garfin SR, et al. Sacral colpopexy followed by refractory Candida albicans osteomyelitis and discitis requiring extensive spinal surgery. Obstet Gynecol. 2012;120(2 Pt 2):464-8.

41. Stepanian AA, Miklos JR, Moore RD, et al. Risk of mesh extrusion and other mesh-related complications after laparoscopic sacral colpopexy with or without concurrent laparoscopic-assisted vaginal hysterectomy: experience of 402 patients. J Minim Invasive Gynecol. 2008;15:188-96.

42. Kohli N, Walsh PM, Roat TW, et al. Mesh erosion after abdominal sacrocolpopexy. Obstet Gynecol. 1998;92:999-1004.

43. Arsene E, Giraudet G, Lucot JP, et al. Sacral colpopexy: long-term mesh complications requiring reoperation(s). Int Urogynecol $J$. 2015;26:353-8.

44. Quiroz LH, Gutman RE, Fagan MJ, et al. Partial colpocleisis for the treatment of sacrocolpopexy mesh erosions. Int Urogynecol J Pelvic Floor Dysfunct. 2008;19:261-6.

45. South MM, Foster RT, Webster GD, et al. Surgical excision of eroded mesh after prior abdominal sacrocolpopexy. Am J Obstet Gynecol. 2007;197:615.e1-5.

46. Mattox TF, Stanford EJ, Varner E. Infected abdominal sacrocolpopexies: diagnosis and treatment. Int Urogynecol J Pelvic Floor Dysfunct. 2004;15:319-23.

47. Buechel M, Tarr ME, Walters MD. Vaginal apical pain after sacrocolpopexy in absence of vaginal mesh erosion: a case series. Female Pelvic Med Reconstr Surg. 2016;22:e8-e10.

48. Kwon SY, Brown S, Hibbeln J, et al. Conservative management of pelvic abscess following sacrocolpopexy: a report of three cases and review of the literature. Int Urogynecol J. 2017;28:875-9.

49. Govier FE, Kobashi KC, Kozlowski PM, et al. High complication rate identified in sacrocolpopexy patients attributed to silicone mesh. Urology. 2005;65:1099-103.

50. Oliver JL, Chaudhry ZQ, Medendorp AR, et al. Complete excision of sacrocolpopexy mesh with autologous fascia sacrocolpopexy. Urology. 2017;106:65-9.

51. Muller PC, Berchtold C, Kuemmerli C, et al. Spondylodiscitis after minimally invasive recto- and colpo-sacropexy: report of a case and systematic review of the literature. J Minim Access Surg. 2020;16:5-12.

52. NICE guidance - urinary incontinence and pelvic organ prolapse in women: management: (C) NICE (2019) urinary incontinence and pelvic organ prolapse in women: management. BJU Int. 2019;123(5):777-803.

53. Deffieux X, de Tayrac R, Huel C, et al. Vaginal mesh erosion after transvaginal repair of cystocele using Gynemesh or Gynemesh-Soft in 138 women: a comparative study. Int Urogynecol J. 2007;18:73-9.

54. MacDonald S, Terlecki R, Costantini E, et al. Complications of transvaginal mesh for pelvic organ prolapse and stress urinary incontinence: tips for prevention, recognition and management. Eur Urol Focus. 2016;2:260-7.

55. Warembourg S, Labaki M, de Tayrac R, et al. Reoperations for mesh-related complications after pelvic organ prolapse repair: 8year experience at a tertiary referral center. Int Urogynecol $\mathrm{J}$. 2017;28:1139-51.

56. Lee D, Chang J, Zimmern PE. Iatrogenic pelvic pain: surgical and mesh complications. Phys Med Rehabil Clin N Am. 2017;28(3): 603-19.

57. Miklos JR, Chinthakanan O, Moore RD, et al. The IUGA/ICS classification of synthetic mesh complications in female pelvic floor reconstructive surgery: a multicenter study. Int Urogynecol J. 2016;27:933-8.

58. Feiner B, Maher C. Vaginal mesh contraction: definition, clinical presentation, and management. Obstet Gynecol. 2010;115(2 Pt 1): 325-30.

59. Ridgeway B, Walters MD, Paraiso MF, et al. Early experience with mesh excision for adverse outcomes after transvaginal mesh placement using prolapse kits. Am J Obstet Gynecol. 2008;199(6): 703.e1-7.

60. Jeffery ST, Nieuwoudt A. Beyond the complications: medium-term anatomical, sexual and functional outcomes following removal of trocar-guided transvaginal mesh. A retrospective cohort study. Int Urogynecol J. 2012;23(10):1391-6.

61. Firoozi F, Ingber MS, Moore CK, et al. Purely transvaginal/perineal management of complications from commercial prolapse kits using a new prostheses/grafts complication classification system. J Urol. 2012;187(5):1674-9.

62. Hokenstad ED, El-Nashar SA, Blandon RE, et al. Health-related quality of life and outcomes after surgical treatment of complications from vaginally placed mesh. Female Pelvic Med Reconstr Surg. 2015;21(3):176-80.

63. Lee D, Dillon B, Lemack G, et al. Transvaginal mesh kits-how "serious" are the complications and are they reversible? Urology. 2013;81(1):43-8.

64. Skala CE, Renezeder K, Albrich S, et al. Mesh complications following prolapse surgery: management and outcome. Eur J Obstet Gynecol Reprod Biol. 2011;159(2):453-6.

65. Toozs-Hobson P, Cardozo L, Hillard T. Managing pain after synthetic mesh implants in pelvic surgery. Eur J Obstet Gynecol Reprod Biol. 2019;234:49-52.

66. Sancak EB, Avci E, Erdogru T. Pudendal neuralgia after pelvic surgery using mesh: case reports and laparoscopic pudendal nerve decompression. Int J Urol. 2016;23(9):797-800.

67. ACOG Practice Committee Opinion no. 513: vaginal placement of synthetic mesh for pelvic organ prolapse. Obstet Gynecol. 2011;118(6):1459-64.

68. 11th Edition of Educational Objectivs: Core Curriculum in Obstetrics and Gynecology. https://www.acog.org/About-ACOG/ ACOG-Departments/CREOG/CREOG-Search/CREOG-11thEdition-of-Educational-Objectives?IsMobileSet=false. Accessed May 6, 2019.

69. Blandon RE, Gebhart JB, Trabuco EC, et al. Complications from vaginally placed mesh in pelvic reconstructive surgery. Int Urogynecol J Pelvic Floor Dysfunct. 2009;20(5):523-31.

70. Dallas KB, Trimble R, Rogo-Gupta L, et al. Care seeking patterns for women requiring a repeat pelvic organ prolapse surgery due to native tissue repair failure compared to a mesh complication. Urology. 2018;122:70-5.

71. Balachandran A, Duckett J. What is the role of the multidisciplinary team in the management of urinary incontinence? Int Urogynecol J. 2015;26(6):791-3.

Publisher's note Springer Nature remains neutral with regard to jurisdictional claims in published maps and institutional affiliations. 